\title{
Dietary protein influences on regulation of Haemonchus contortus populations in Dorsimal lambs
}

\begin{abstract}
The influence of dietary protein supplementation upon resistance to haemonchosis was examined in Dorsimal (Polled Dorset x Malin) lambs offered two levels of protein. Lambs were offered either a complete basal ruminant diet $(15 \%$ crude protein $(\mathrm{CP}))$ or the same diet supplemented with fish meal as a source of rumen bypass protein $(19 \% \mathrm{CP})$. Lambs from each dietary treatment group were given either a 7-week trickle infection with Haemonchus contortus infective larvae (L3) or remained uninfected. All lambs were drenched with anthelmintic at week 8 post-infection (PI), challenged with a single dose of $5000 \mathrm{H}$. contortus L3 one week later, and killed 14 days post-challenge (PC). Lambs on the supplemented diet that were trickle infected showed a significant reduction in egg output. Supplementation and previous infection did not affect either growth rate, worm burden, worm development or haematological parameters. There was a trend for enhanced growth among supplemented non-infected lambs in comparison to lambs which received the basal ration.
\end{abstract}

Keyword: Dietary protein; Dorsimal lambs; Haemonchus contortus 